\title{
Analytical Potential Energy Curve of Fullerenes Dimers Using the Girifalco's Potential
}

lribeiro@ueg.br

\author{
João Paulo de Araújo Martins ${ }^{1}(\mathrm{IC})$, Luciano Ribeiro ${ }^{2 *}(\mathrm{PQ})$
}

1 - Câmpus Anápolis de Ciências Exatas e Tecnológicas - Henrique Santillo - UEG, BR 153 N. 3105 , Anápolis - GO, Brazil

Keywords: Fullerenes, Dunham, DVR, Girifalco Model

\section{Introduction}

Discovered in 1985 by Kroto and co-authors ${ }^{1}$, fullerenes have been the studied from both experimental and theoretical standpoints. Fullerenes, also called Buckminsterfullerenes, are carbon-based molecules that interact with each other through van der Waals forces. They have been applied in several fields of knowledge, such as acting as optical limiters, superconductors, materials science design, and mainly in nanotechnology. Although fullerenes have shown icosahedral symmetry, their shape can be approximated as a sphere, since the molecules freely rotate.

Furthermore, a configuration composed of a C60 dimer can be considered a diatomic molecule, and just like the bonds between the carbons of the same fullerene, the interactions between $\mathrm{C60}$ dimers stabilize by van der Waals forces. An approximation for a spherical distribution of uniform density of carbon atoms can also be used for these molecules, where the total potential is the sum of the individual potentials of each carbon atom in one molecule interacting with the other.

Taking advantage of this, Girifalco used the Lennard-Jones Potential for the carbon interactions between the molecules and, integrating the surface of this sphere with 60 carbon atoms, obtained the expression known as Girifalco's Potential.

In the paper of L. Girifalco ${ }^{2}$, an analytical form, now known as Girifalco's Potential, was proposed, which was constructed by integrating a surface of a sphere containing 60 carbon atoms. Girifalco started from the proposal of the Lennard-Jones potential and obtained the expression described by Eq. (1)

$$
\begin{aligned}
V(\alpha, \beta, s)=- & \alpha\left[\frac{1}{s(s-1)^{3}}+\frac{1}{s(s+1)^{3}}-\frac{2}{s^{4}}\right] \\
+ & \beta\left[\frac{1}{s(s-1)^{9}}+\frac{1}{s(s+1)^{9}}-\frac{2}{s^{10}}\right],
\end{aligned}
$$

where, $s=r / 2 a, r$ being the separation between the center of dimer, radius a, $\alpha=$ $3600 A / 768 a^{6}$, and $\beta=3600 B / 368640 a^{12} . A$ and $B$ are parameters and $r$ is the distance between the atoms.

\section{Methodology}

This proposal allows us to investigate the properties of a C60 molecule. Through Girifalco's Potential, we can analytically describe the potential energy curve (PEC) of the C60 dimer. The PEC will be attained from the electronic energies of the van der Waals interaction obtained by ab initio calculations. Through the nonlinear fitting of the parameters of the analytical function and the construction of the PEC, we obtain the constants of forces and spectroscopic constants using Dunham ${ }^{3}$ and Discrete Variable Representation ${ }^{4}$ methods.

\section{Results and Discussions}

The PECs for C60 dimer are shown in Figure 1, where the blue circles are the ab initio calculated values with $\omega \mathrm{b} 97 \mathrm{x}-\mathrm{d} / 6-31 \mathrm{~g}(\mathrm{~d})$ level of theory as implemented in the Gaussian 
09 suite of program and the blue solid line represent the fitted line and its error fits the adjustment at red trashed line.

The optimized parameters for the analytical Girifalco's potential function of the configurations $\mathrm{C}_{60}$ and $\mathrm{C}_{70}$ dimers are presented in Table 1. Table 2 presents the rovibrational spectroscopic parameters as obtained from both the Dunham and DVR methods. From Table 2 it is evident that both methodologies had comparable performance with negligible differences.

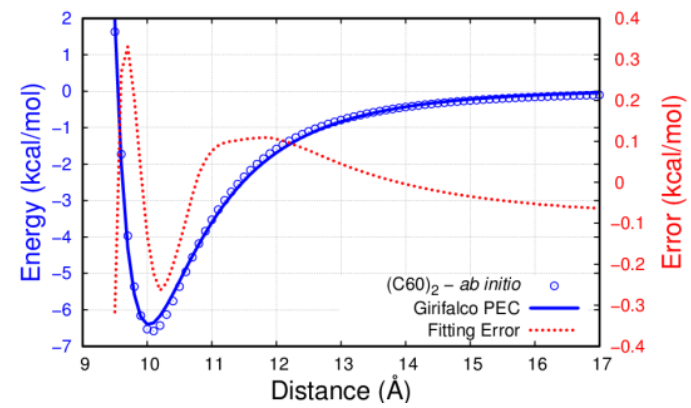

Figura 1 - The fitted Girifalco PEC for fullerene dimer C60. Point-by-point error is also plotted to magnify the overall fitting accuracy in the entire range of internuclear separation.

Table 1 - The parameters of the analytic potential function at Eq. 1.

\begin{tabular}{ccc}
\hline & $\mathrm{C}_{60}$ Dimer & $\mathrm{C}_{70}$ Dimer \\
\hline$\alpha[\mathrm{kcal} / \mathrm{mol}]$ & 1.4714 & 0.6874 \\
$\beta[\mathrm{kcal} / \mathrm{mol}]$ & $4.4411 \times 10^{-3}$ & $5.0978 \times 10^{-4}$ \\
$a[\AA]$ & 3.4502 & 4.1555 \\
$\chi^{2}$ & $0.92 \times 10^{-2}$ & $0.14 \times 10^{-1}$ \\
\hline
\end{tabular}

Table 2 - The derived spectroscopic constants $\left(\mathrm{cm}^{-1}\right)$ for $C_{60}$ and $C_{70}$ dimers.

\begin{tabular}{cccccc}
\hline \multicolumn{2}{c}{$\begin{array}{c}\text { Dimers- } \\
\text { Methods }\end{array}$} & $B_{e} \times 10^{-4}$ & $\omega_{e}$ & $\omega_{e} x_{e} \times 10^{-2}$ & $\alpha_{e} \times 10^{-7}$ \\
\hline \multirow{2}{*}{$\mathrm{C}_{60}$} & Dunham & 3.11 & 26.1 & 8.03 & 3.23 \\
& DVR & - & 26.1 & 8.03 & 5.54 \\
$\mathrm{C}_{70}$ & Dunham & 3.11 & 26.3 & 7.99 & 3.18 \\
& DVR & - & 26.3 & 7.99 & 5.88 \\
\hline
\end{tabular}

\section{Conclusions}

In this study, we have performed adjusting analytical function to describe the potential energy curves of the fullerenes dimers by way Girifalco's Potential. We derive Girifalco's analytical function to obtain the system's force constants. The spectroscopic constants $\omega_{\mathrm{e}}, \omega_{\mathrm{e}} x_{\mathrm{e}}, \mathrm{B}_{\mathrm{e}}$ and $\alpha_{\mathrm{e}}$ were obtained through the Dunham and DVR methods. The values obtained with both methods showed high accuracy.

\section{Acknowledgments}

The authors gratefully acknowledge the support given to this study by from UEG.

\section{References}

1. Kroto, H. W., Heath, J. R., O’Brien, S. C., Curl, R. F. \& Smalley, R. E. C60:

Buckminsterfullerene. Nature 318, 162-163 (1985).

2. Girifalco, L. A. Interaction potential for carbon (C60) molecules. J. Phys. Chem. 95, 5370-5371 (1991).

3. Dunham, J. L. The Energy Levels of a Rotating Vibrator. Phys. Rev. 41, 721-731 (1932).

4. Soares Neto, J. J. \& Costa, L. S. Numerical Generation of Optimized Discrete Variable Representations. Brazilian J. Phys. 28, 1-11 (1998). 